ARTICLE

\title{
Functional annotation of rare gene aberration drivers of pancreatic cancer
}

Yiu Huen Tsang ${ }^{1, \star}$, Turgut Dogruluk ${ }^{1, \star}$, Philip M. Tedeschi ${ }^{2}$, Joanna Wardwell-Ozgo ${ }^{1}$, Hengyu Lu ${ }^{1}$, Maribel Espitia $^{3}$, Nikitha Nair ${ }^{1}$, Rosalba Minelli ${ }^{1}$, Zechen Chong ${ }^{4}$, Fengju Chen ${ }^{5}$, Qing Edward Chang ${ }^{6}$, Jennifer B. Dennison ${ }^{3}$, Armel Dogruluk', Min Li ${ }^{7}$, Haoqiang Ying ${ }^{6}$, Joseph R. Bertino ${ }^{2}$, Marie-Claude Gingras ${ }^{1,8}$, Michael Ittmann', John Kerrigan ${ }^{2}$, Ken Chen ${ }^{4}$, Chad J. Creighton ${ }^{5,10}$, Karina Eterovic ${ }^{3}$, Gordon B. Mills ${ }^{3} \&$ Kenneth L. Scott ${ }^{1,5}$

As we enter the era of precision medicine, characterization of cancer genomes will directly influence therapeutic decisions in the clinic. Here we describe a platform enabling functionalization of rare gene mutations through their high-throughput construction, molecular barcoding and delivery to cancer models for in vivo tumour driver screens. We apply these technologies to identify oncogenic drivers of pancreatic ductal adenocarcinoma (PDAC). This approach reveals oncogenic activity for rare gene aberrations in genes including NAD Kinase $(N A D K)$, which regulates $\operatorname{NADP}(\mathrm{H})$ homeostasis and cellular redox state. We further validate mutant NADK, whose expression provides gain-of-function enzymatic activity leading to a reduction in cellular reactive oxygen species and tumorigenesis, and show that depletion of wild-type NADK in PDAC cell lines attenuates cancer cell growth in vitro and in vivo. These data indicate that annotating rare aberrations can reveal important cancer signalling pathways representing additional therapeutic targets.

\footnotetext{
${ }^{1}$ Department of Molecular and Human Genetics, Baylor College of Medicine, One Baylor Plaza, Houston, Texas 77030, USA. ${ }^{2}$ Rutgers Cancer Institute of New Jersey, 195 Little Albany Street, New Brunswick, New Jersey 08903, USA. ${ }^{3}$ Department of Systems Biology, University of Texas M.D. Anderson Cancer Center, 1515 Holcombe Boulevard, Houston, Texas 77030, USA. ${ }^{4}$ Department of Bioinformatics and Computational Biology, University of Texas M.D. Anderson Cancer Center, 1515 Holcombe Boulevard, Houston, Texas 77030, USA. ${ }^{5}$ Dan L. Duncan Cancer Center, Baylor College of Medicine, One Baylor Plaza, Houston, Texas 77030, USA. ${ }^{6}$ Department of Genomics Medicine, University of Texas M.D. Anderson Cancer Center, 1515 Holcombe Boulevard, Houston, Texas 77030, USA. ${ }^{7}$ Department of Medicine, The University of Oklahoma Health Sciences Center, Oklahoma City, Oklahoma 73104, USA. ${ }^{8}$ Human Genome Sequencing Center, Baylor College of Medicine, One Baylor Plaza, Houston, Texas 77030, USA. ${ }^{9}$ Department of Pathology and Immunology, Baylor College of Medicine, One Baylor Plaza, Houston, Texas 77030, USA. ${ }^{10}$ Department of Medicine, Baylor College of Medicine, One Baylor Plaza, Houston, Texas 77030, USA. * These authors contributed equally to this work. Correspondence and requests for materials should be addressed to K.L.S. (email: kls1@bcm.edu).
} 
arge-scale efforts such as The Cancer Genome Atlas and International Cancer Genome Consortium (ICGC) are generating a compendium of genomic aberrations across major cancer types. Such studies are revealing the complexity of cancer genomes, which contain pathogenic driver aberrations and many more biologically neutral passenger events. While most cancers acquire one or more well-studied, high-frequency drivers, much less is known about which and how the abundant lowfrequency variants contribute to tumour progression. Experimental assessment of low-frequency aberrations, in both undercharacterized genes or among the long 'tail' mutations observed in known cancer genes, is difficult, given their large number and the fact that they may either directly drive tumour progression or indirectly influence tumour behaviour through modifying activities of other driver aberrations. Moreover, distinguishing driver events from passengers is further complicated by the fact that driver activity is shaped by the biological context of a given cancer, including its tissue type, microenvironment and other host determinants. Identifying rare driver aberrations therefore requires robust pipelines that allow context-specific functional prioritization of the thousands of potential targets emerging from Next Generation Sequencing (NGS) data.

Here we describe a gain-of-function (GOF) screening platform enabled by a High-Throughput Mutagenesis and Molecular Barcoding (HiTMMo $\bar{B})$ method allowing cost-effective, rapid modelling and expression of molecularly barcoded mutant gene variants into open reading frame (ORF) expression clones for pooled functional screens. HiTMMoB permits annotation of mutation-activated oncogenes, whose identification is particularly desirable, given the efficacy of antibody and small molecule inhibitor therapies that may be tailored towards such proteins. Our approach complements gene depletion and knockout strategies by RNA interference (RNAi) and CRISPR/Cas9-based platforms, respectively, which have been tremendously successful for identifying and validating tumour suppressor genes and other genetic dependencies ${ }^{1,2}$. HiTMMoB builds on previous overexpression screen-based studies designed to identify wild-type ORFs whose expression promotes tumorigenesis ${ }^{3,4}$, drug resistance ${ }^{5}$ and other cancer cell phenotypes such as anchorageindependent growth ${ }^{6-8}$ among others. Such screens are made possible through efforts by the ORFeome collaboration (http:// www.orfeomecollaboration.org/; refs 9-11) and others ${ }^{12}$ who have systematically cloned and sequenced-validated a large majority of expressed human transcripts.

In this study we use HiTMMoB to identify low-frequency mutational events in pancreatic ductal adenocarcinoma (PDAC) that serve as drivers or effectors for recurrent drivers such as
KRAS, a proto-oncogene activated in $>90 \%$ of PDAC cases ${ }^{13}$. We employed HiTMMoB to build PDAC aberration libraries, whose compositions were based on individual PDAC patient genomes and computational selection, to identify rare oncogenic events that, when expressed in a human pancreatic cell model and implanted into mice, promotes in vivo tumour formation. This approach revealed potent activity for aberrations in genes including $N A D$ Kinase $(N A D K)$ that regulates $\mathrm{NADP}(\mathrm{H})$ homeostasis and cellular redox state. We show that mutant $N A D K$ provides GOF enzymatic activity, leading to a reduction in cellular reactive oxygen species (ROS) and tumorigenesis. In addition, we show that depletion of wild-type NADK in PDAC cell lines attenuated cancer cell growth in vitro and in vivo. We conclude that barcode (BC)-assisted, GOF screening has the potential to have an impact on the field of cancer target discovery as a whole, particularly with respect to identifying low-frequency somatic events by complementing structural characterizations of cancer genomes with functional annotation of gene aberrations identified from tumour sequencing programmes.

\section{Results}

HiTMMoB method. Successful translation of NGS data requires knowledge on which DNA aberrations represent actionable events, either for development or re-positioning of approved agents to target their activated pathways. To circumvent technical bottlenecks related to scaled construction of aberration expression clones, we developed a cost-effective HiTMMoB method (Fig. 1a) that employs: (1) two common PCR primers (P1/P2) flanking the ORF gene target and a third primer containing the desired mutation (PM), (2) a three-stage PCR reaction, whose design incorporates differential $\mathrm{P} 1 / \mathrm{P} 2$ and $\mathrm{PM}$ melting temperatures ${ }^{14}$ allowing simultaneous use of all three primers with target ORFs available from the ORFeome collaboration ${ }^{11}$ and others and (3) Gateway (Life Technologies)-mediated recombineering of the mutant PCR product into compatible destination vectors. HiTMMoB permits simplified and sequential completion of mutagenesis and ORF cloning without intermediate DNA gel purification steps, making it cost-effective and scalable to a 96well format leading to individual bacterial colonies carrying expression-ready mutant ORFs in less than $48 \mathrm{~h}$. Shown in Supplementary Data 1 are representative ORF mutagenesis attempts (missense mutations and small insertions/deletions) for 175 reactions, where HiTMMoB achieved 99\% mutation success (173 mutant ORFs constructed in a single attempt) to target ORFs ranging from 303 to 4,863 bp in length and 38 to $74 \%$ in DNA G/C content. In this example, HiTMMoB achieved 76\%
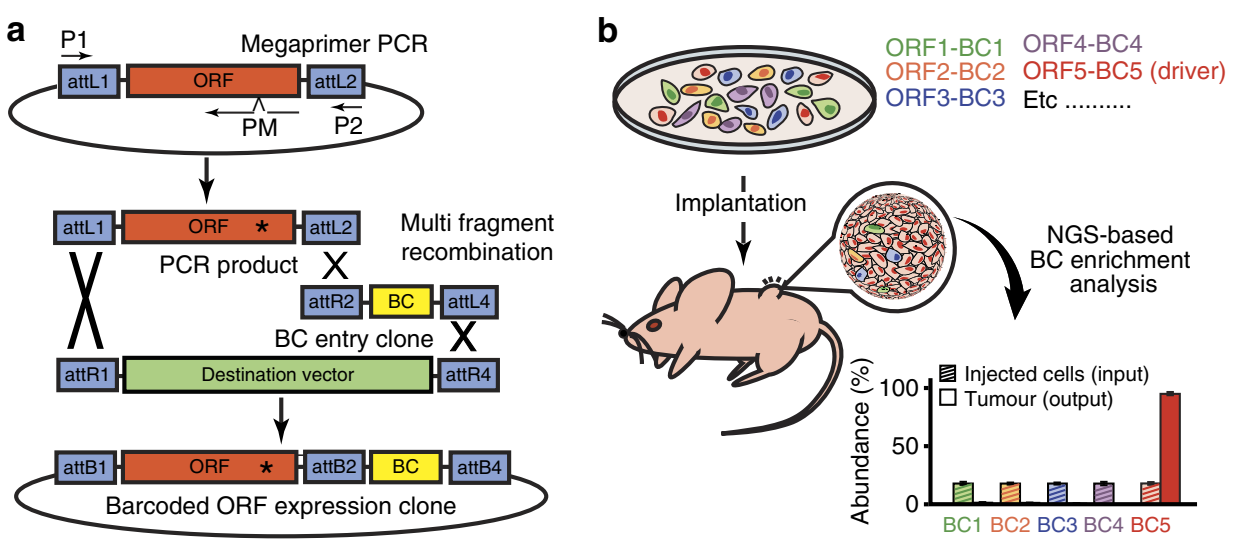

Figure 1 | Functional annotation of cancer gene aberration HiTMMoB. Illustration of (a) HiTMMoB-mediated mutagenesis and molecular barcoding, and (b) barcode enrichment analysis. See text for details. BC, barcode; P1/P2/PM, PCR primers; attL/R/B, recombination sequences. 

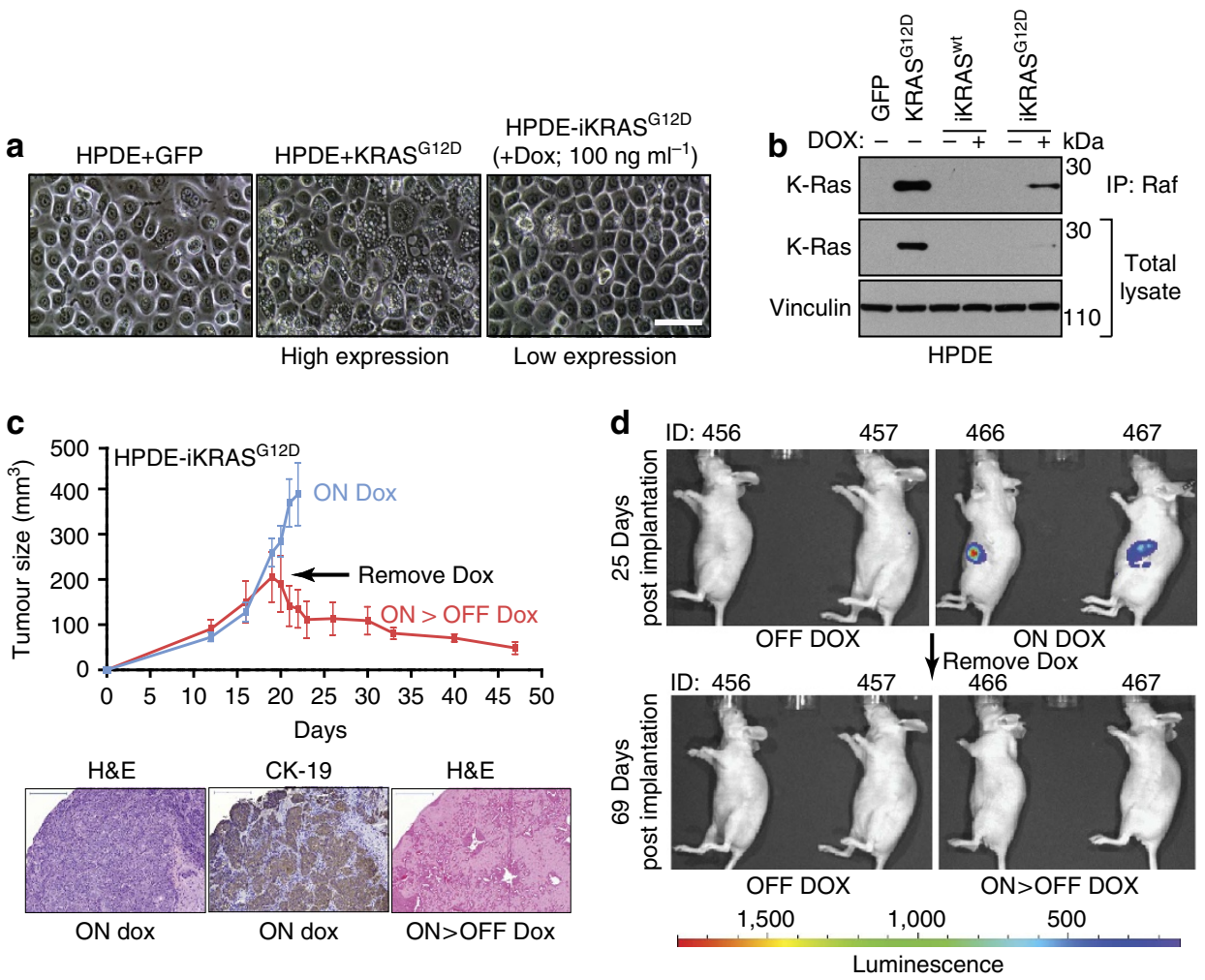

Figure 2 | HPDE-iKRAS $\mathbf{G 1 2 D}^{\mathbf{G}}$ cell model. (a) HPDE cells expressing (left) GFP control and (middle) high levels of KRAS G12D. (Right) HPDE-iKRAS G12D cells treated with Dox. Scale bar, $5 \mu \mathrm{m}$. (b) Immunoblot analysis of parental HPDE cells expressing GFP or high levels of KRAS G12D (leftmost two lanes) and the indicated HPDE-inducible cell lines. Protein lysates were immunoprecipitated (IP) with antibody against RAF as an indicator of Ras activation. (c,d) HPDE-iKRAS $S^{G 12 D}$ xenograft growth (mean tumour volume, error bars denote s.d.) of biological replicates $(N=7$ bilaterally injected animals $=14$ tumours per group) in the presence of Dox (On Dox) and following removal of Dox (On> Off Dox) implanted subcutaneously (c) and orthotopically into the pancreas (d). Representative haematoxylin and eosine and CK-19 staining of the indicated tumours shown at bottom. Scale bars, $200 \mu \mathrm{m}$.

efficiency based on the number of mutation-positive ORFs isolated from multiple single bacterial colonies resulting from each mutagenesis reaction. The fact that HiTMMoB achieves $\sim 75 \%$ efficiency per reaction allows straightforward clonal isolation of individual ORFS, which we fully sequence-verify before use in downstream applications. For example, as shown in Supplementary Data 1, an average of 3.8 colonies per each of the 175 mutagenesis reactions was sufficient to obtain 173 of 175 desired mutant clones.

HiTMMoB also incorporates co-integration of individual DNA 'BCs' with wild-type or mutant ORFs into destination vectors using multifragment DNA recombination (Fig. 1a). The barcoding strategy relies on a panel of individual $\mathrm{BC}$ entry clones, each designed to contain a unique 24-bp $\mathrm{BC}$ designed to be an isothermal set so that individual $\mathrm{BCs}$ are recombined into destination vectors only in the presence of wild-type or mutant ORFs. This flexible barcoding approach allows rapid use and recycling of $\mathrm{BCs}$ during ORF library preparation in diverse destination vectors, providing barcoded expression clones within $24 \mathrm{~h}$ following recombination, thus eliminating the need to $\mathrm{BC}$ entire ORF collections before use. This barcoding flexibility is especially useful when constructing allelic series of an individual gene (see Supplementary Data 1, for example), as each variant of the series can be simultaneously barcoded without any need of inserting BCs into every vector as typically done using conventional DNA ligation methods. Each BC serves as a surrogate identifier for its associated ORF, thus permitting individual ORF detection by NGS. Individual ORF enrichment (positive selection) or depletion (negative selection) among pooled ORFs can be determined by comparing the number of individual $\mathrm{BC}$ reads within experimental pools as a ratio of each $\mathrm{BC}$ to the total number of total $\mathrm{BC}$ reads per sequenced amplicon. For example, in the case of in vivo tumour driver enrichment screens (Fig. 1b) as used here, ORF-driven tumours (output) will be positively enriched for driver-associated $\mathrm{BCs}$ (BC5) and lose those with no role in progression (that is, passengers, BC1-4) compared with injected cells (input) carrying all barcoded ORFs.

Model construction for PDAC driver discovery. We first sought to apply HiTMMoB to identify novel, rare aberration drivers of PDAC. In an effort to model the genetic context of early PDAC, we integrated a doxycycline (Dox)-regulatable KRAS $S^{G 12 D}$ transgene $\left(i K R A S^{G 12 D}\right)$ into previously engineered non-transformed, human pancreatic ductal epithelial (HPDE) cells previously engineered $^{15,16}$ with the E6 and E7 proteins of the HPV16 virus to emulate loss of p53 and inactivation of the Rb pathway, respectively ${ }^{15,16}$. Parental HPDE cells expressing high levels of $K R A S^{G 12 D}$ underwent significant morphological changes and loss of proliferative capacity (Fig. 2a) consistent with oncogeneinduced senescence ${ }^{17}$. In contrast, HPDE- $i K R A S^{G 12 D}$ cells treated with Dox appeared to be morphologically similar to parental cells (Fig. 2a) owing to the titratable and near-physiological level of KRAS protein (Fig. 2b) that reduces the potential for KRAS ${ }^{G 12 D_{-}}$ mediated senescence. RAF-immunoprecipitation analysis (Fig. 2b) indicated a significant KRAS activity in HPDEiKRAS ${ }^{G 12 D}$ cells treated with Dox compared with untreated and wild-type (HPDE-iKRAS ${ }^{w t}$ ) control cells. RNA profiling of 
a

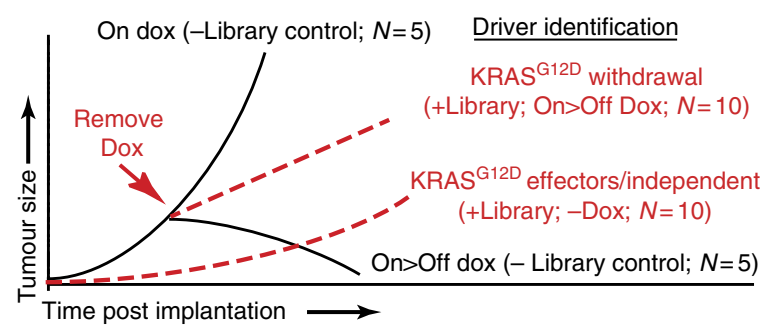

C

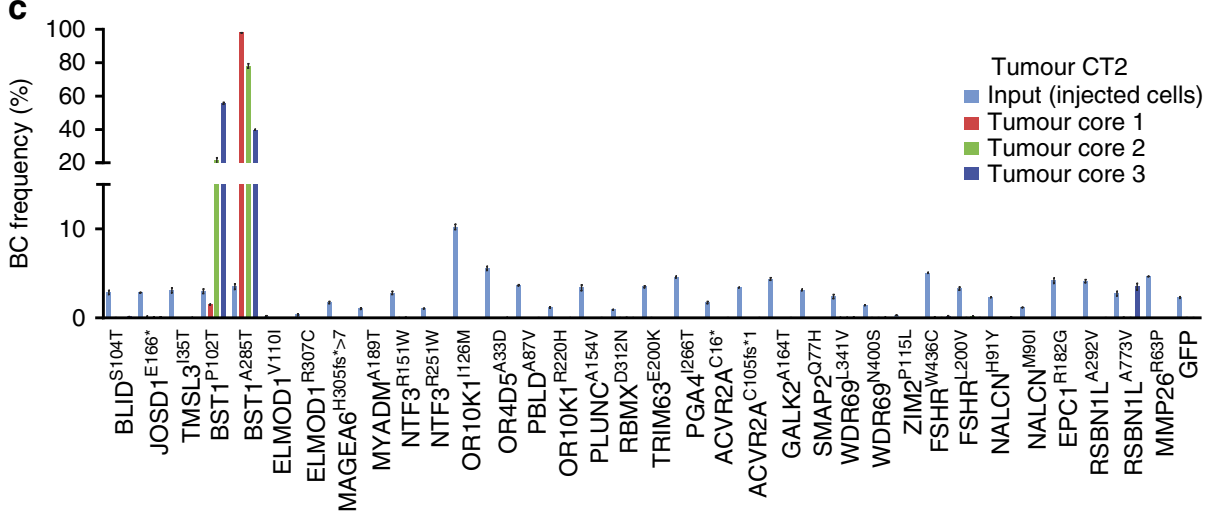

b -Library controls

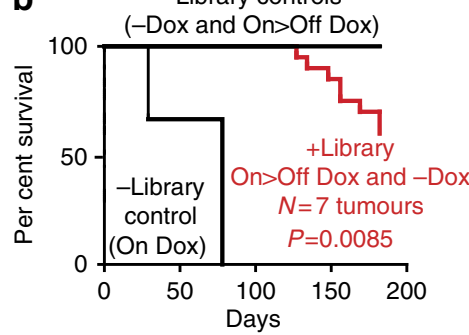

d

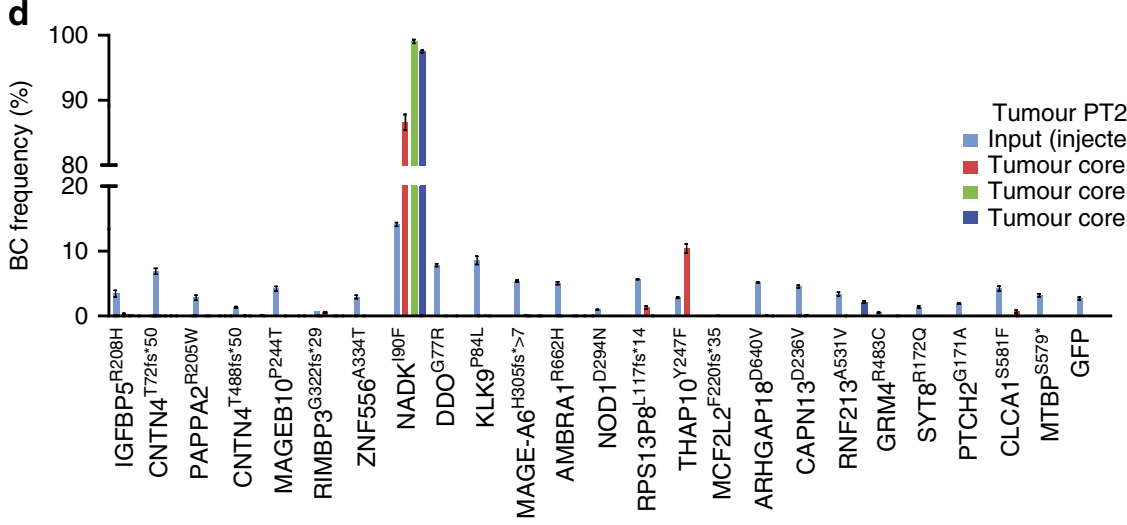

Figure 3 | In vivo functional screening for aberration drivers of PDAC. (a) Screen schematic. HPDE-iKRAGG12D cells expressing GFP only ( - Library control; $N=5$ each for On Dox and On> Off Dox cohorts) or candidate-barcoded ORFs plus GFP were pooled ( + Library; $N=10$ each for On Dox and On $>$ Off Dox cohorts) and injected into athymic mice subcutaneously. Mice were divided into On Dox, Off Dox ( - Dox) or transitioned On to Off Dox (ON > OFF Dox) cohorts to look for KRAS-dependent and KRAS-independent drivers. (b) Kaplan-Meier survival plot for computationally informed screen cohorts as indicated in a. $P$ value calculated by log-rank test. (c,d) Barcode enrichment analysis of representative tumour (c) CT2 and (d) PT2. Data post normalization to total reads (sum of reads of barcodes + GFP) are shown as mean \pm s.d. of sequencing technical replicates ( $N=3$ for each input and core).

HPDE-iKRAS $S^{G 12 D}$ cells revealed widespread changes in the presence of Dox $(2,824$ gene probes; fold change $>2.0$ for onDox versus off-Dox, $P<0.01, t$-test) compared with HPDE-iGFP and $-i K R A S^{w t}$ cells (Supplementary Fig. 1a and Supplementary Data 2). Within the KRAS ${ }^{G 12 D}$-induced genes, the Molecular Signatures Database identified 'Bild_Hras_Oncogenic_Signature' as a top-enriched gene set $\left(P=3.76 \times 10^{-29}\right.$, one-sided Fisher's exact test; Supplementary Data 3). Upregulation of Ras signalling in Dox-treated HPDE-iKRAS G12D cells was also supported by a significant overlap of KRAS dependency signature genes reported by Settleman and colleagues ${ }^{18}\left(P=7.0 \mathrm{E}^{-22}\right.$, one-sided Fisher's exact test) and Reverse-Phase Protein Array (RPPA) analysis (Supplementary Fig. 1b).

We next evaluated HPDE-iKRAS G12D cells for oncogenic growth. Placing the cells in anchorage-independent growth conditions in the presence of Dox led to a significant $(P=0.0001$, $t$-test) increase in colony formation compared with off-Dox control cells (Supplementary Fig. 1c), and implanting HPDE-iKRAS $S^{G 12 D}$ cells subcutaneously into the flanks of athymic mice $(N=14)$ led to a rapid formation of poorly differentiated, CK-19-positive carcinomas $(N=12)$ in animals on Dox compared with no tumours observed in animals maintained off Dox (Fig. 2c). Randomizing on-Dox animals into an off-Dox cohort led to complete tumour regression, revealing remnants of pre-existing tumour with underlying vasculature in co-injected Matrigel (BD Biosciences) matrix (Fig. 2c). We next modified HPDE-iKRAS ${ }^{\text {G12D }}$ cells by including a constitutively expressing allele of firefly luciferase whose expression enables live animal serial imaging following injection of D-luciferin. Similar to the subcutaneous tumour growth studies above, HPDE-iKRAS ${ }^{G 12 D}$ cells progressed to tumours within 2 weeks post implantation into the pancreas of mice maintained on Dox diet, and these tumours regressed below 
the limits of detection within 45 days following removing Dox (Fig. 2d). In summary, we derived and characterized an HPDEiKRAS ${ }^{G 12 D}$ cell model for use in primary screens to identify PDAC gene aberrations that drive in vivo tumour growth.

In vivo pooled screening for aberration drivers of PDAC. We next resourced PDAC tumour sequencing data from the ICGC ${ }^{13}$, whose analyses by three separate statistical methods (Fisher's combined $P$ value test, likelihood ratio test and convolution test) identified significantly mutated genes containing non-silent mutations. We first chose the 59 significantly mutated genes ranked in the top 100 by all three statistical methods following exclusion of known drivers KRAS, TP53, CDKN2A, SMAD4, ATM and ARIDIA (Supplementary Table 1). For the remaining 53 gene candidates, we employed HiTMMoB to engineer 73 unique, non-silent mutations into $41 \mathrm{ORFs}$ after excluding aberrations where ORFs were unavailable. In total, we were able to construct 70 (95.9\%) barcoded aberration clones for entry into the functional screening platform.

To examine oncogenic activity of these computationally selected aberrations, HPDE-iKRAS ${ }^{G 12 D}$ cells stably transduced with barcoded control (green fluorescent protein (GFP)) and aberration lentivirus were pooled into two populations, each implanted into a single flank of 20 athymic mice (total 40 animals, Fig. 3a). For each of the two screen cohorts, 10 animals were maintained off Dox diet to identify drivers that function downstream or independent of $K R A S^{G 12 D}$. The remaining 10 animals for each screen cohort were placed on Dox diet to promote $K R A S^{G 12 D}$ driven tumorigenesis, followed by transitioning animals with palpable tumours $\left(200 \mathrm{~mm}^{3}\right)$ off-Dox (denoted On $>$ Off Dox) to identify events that enable tumour escape from Dox withdrawal. All animals were monitored for tumour formation and growth as compared with reference control mice (Fig. 3a). Screening using this approach identified seven tumours (CT1-7; $N=6$ in mice offdox, $N=1$ in mice On $>$ Off Dox) $18-26$ weeks post injection (Fig. 3b). To assess ORF enrichment in those seven tumours, we quantitated ORF BC reads from genomic DNA (gDNA) isolated from tumour (output) and injected cells (input) using an Ion Torrent Personal Genome Machine (PGM; 66 multiplexed samples: three input gDNA replicates + three gDNA replicates from three individual cores for each of the seven tumours; replicate $=$ technical replicate PGM amplicon library preparations). Analysis of the 21 cores taken from CT1-7 revealed a strong enrichment for variants of Bone Marrow Stromal Cell Antigen 1, BST1 (BST1 ${ }^{P 102 T}$ and BST1 ${ }^{A 285 T}$ ) versus input control (representative tumour CT2 shown in Fig. $3 \mathrm{c}$ and Supplementary Fig. 2). We observed minimal variation across technical replicate for amplicon PGM library preparations (see error bars, Fig. 3c). Notably, individual cores from CT6 and CT7 exhibited enrichment for BST1 variants along with a second ORF (WDR69 ${ }^{L 341 V}$; Supplementary Fig. 2), indicating an ability to identify multiple driver ORFs acting alone or in combination in a given tumour. Similarly, tumours CT5 and CT6, which demonstrated significant necrosis at the time of animal necropsy, thus increasing sequencing deviation across replicate PGM libraries, revealed high-level enrichment of BST1 ${ }^{P 102 T}$ and BST1 ${ }^{A 285 T}$ as well as coenrichment for ELMO/CED-12 Domain Containing 1, $E L M O D 1^{R 307 C}$, in all tumour cores (Supplementary Fig. 2).

In addition to our computational approach towards candidate selection, we also took an unbiased approach by randomly choosing four patients sequenced by the ICGC ${ }^{13}$ whose tumours contained activating and inactivating mutations in KRAS and TP53, respectively. We then engineered aberration (missense mutations and small insertions/deletions; $N=24$ ) expression constructs for all events in the four patients in genes represented in our ORF collection (Supplementary Table 1). Screening using the same in vivo approach (Fig. 3a) identified four tumours (PT1-4) in the library-injected cohorts $(N=3$ in mice off Dox, $N=1$ in mice On $>$ Off Dox) at 21 weeks post injection. BC enrichment analysis of 40 multiplexed samples (four replicates for Input gDNA + three gDNA replicates from three individual cores for each four tumours) revealed a high-level enrichment for mutant NADK (NADK ${ }^{190 F}$; average $94 \%$ enrichment) in three out of four tumours (PT2-4) as well as a low-level enrichment (11\%) for THAP Domain Containing 10, THAP10 ${ }^{Y 247 F}$, in a single core of PT2 (Fig. 3d and Supplementary Fig. 3). Notably, tumour PT4 demonstrated significant necrosis at the time of necropsy, and sequencing analysis revealed co-enrichment for Insulin-Like Growth Factor Binding Protein 5, IGFBP5 ${ }^{R 208 H}$, in two cores (C1 and $\mathrm{C} 2)$ taken from the necrotic region of the tumour compared with a non-necrotic core (C3) fully enriched for $N A D K^{I 90 F}$ (C1, 99\%; Supplementary Fig. 3). Finally, analysis of PT1 (Supplementary Fig. 3) indicated complete enrichment of Melanoma Antigen Family A-6, MAGE-A6 ${ }^{C \triangle 304}$, across all three cores analysed for this tumour. In summary, this first use of HiTMMoB for primary screening of ICGC data prioritized driver aberrations with greatest enrichment for variant ORFs encoding BST1, MAGEA6 and NADK (Supplementary Table 2).

NADK ${ }^{190 F}$ exhibits GOF activity in PDAC. Our primary screen revealed high enrichment for a mutant ORF encoding NADK $\left(N A D K^{190 F}\right)$, which was identified by the ICGC $^{13}$ to be mutated in a single tumour (PCSI0023_T). NADK catalyses the conversion of cytoplasmic $\mathrm{NAD}^{+}$to $\mathrm{NADP}^{+} / \mathrm{NADPH}$ and contributes to cellular NADPH production ${ }^{19}$. We chose to validate NADK driver activity, given the importance in maintaining the redox state in PDAC 20 , potential druggablity as a kinase and our premise that functionalizing rare mutations, even those unique to a single patient, may inform new modes to activate important signalling pathways. Re-construction and implantation of HPDE cells expressing $N A D K^{I 90 F}$ validated its tumour-initiating activity $(N=8 / 10 ; \quad P=0.0133, \log$-rank test) compared with vector control $(N=0 / 10)$, leading to a significant decrease in overall survival for the $N A D K^{I 90}$ Cohort (Fig. 4a). Wild-type NADK exhibited very weak tumour-initiating activity $(N=4 / 10)$, as three of the four $N A D K^{W T}$ tumours remained growth-static or regressed (Fig. 4a). In contrast, $N A D K^{I 90 F}$-expressing tumours grew to maximal tumour burden and were significantly larger than the static $N A D K^{W T}$-expressing tumours, despite a single $N A D K^{W T}$ outlier tumour (Fig. 4a; Grubb's extreme Studentized deviate outlier test, $P<0.05$ ).

In silico molecular dynamics simulation of the $190 \mathrm{~F}$ mutation based on the crystal structure of dimerized human NADK ${ }^{21}$ indicated a significant $190 \mathrm{~F}$-induced change in the radius of gyration (Fig. 4b), a measure of structure compactness, predicted to have an impact on catalytic activity as observed in other mutant enzymes 22 . Brooks et al. observed that distal mutations can have an impact on correlated motions along the backbone of an enzyme, thereby having an impact on its catalytic activity ${ }^{22}$. Consistent with this finding, simulation trajectory analyses revealed an increase in coupled motions in the I90F variant compared with wild-type (Supplementary Fig. 4). Moreover, plotting the electrostatic potential of the substrate-binding region of NADK revealed a significant change in shape and size of the positive electrostatic potential in the $190 \mathrm{~F}$ variant compared with wild type (Fig. 4c), suggesting that the I90F mutation opens the NADK dimer interface to increase substrate availability, leading to greater kinase activity. Together, these data led us to examine kinase activity by recombinant wild-type and I90F mutant protein (Supplementary Fig. 5). NAD + and ATP enzymatic assays 
a
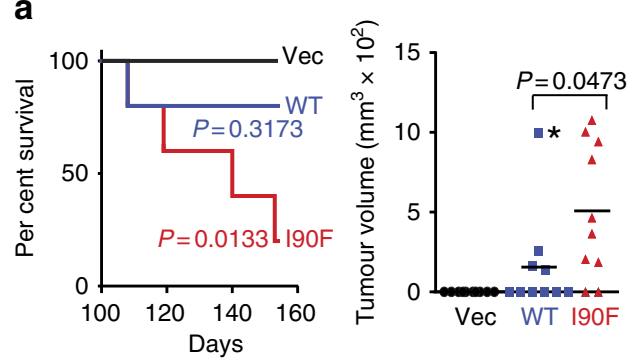

b

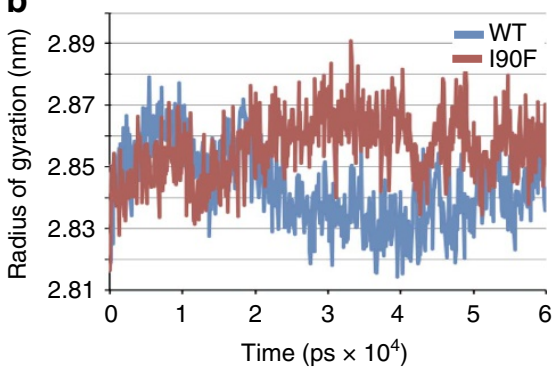

C

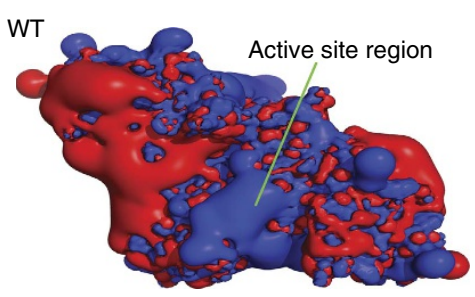

190F

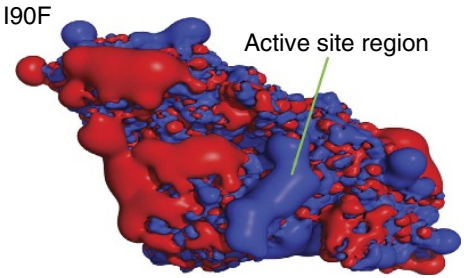

d
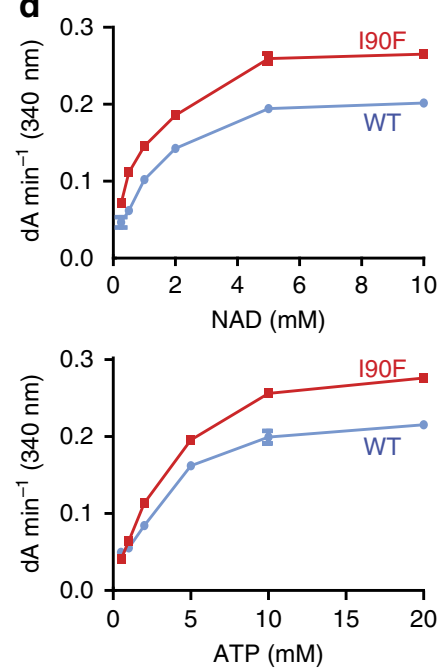

e
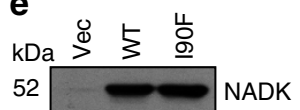

38

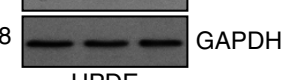

HPDE
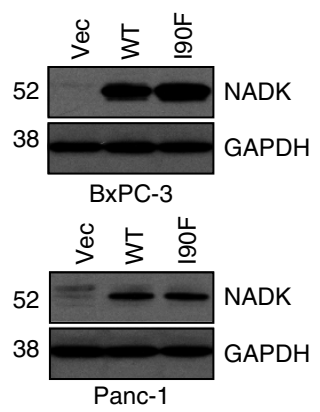
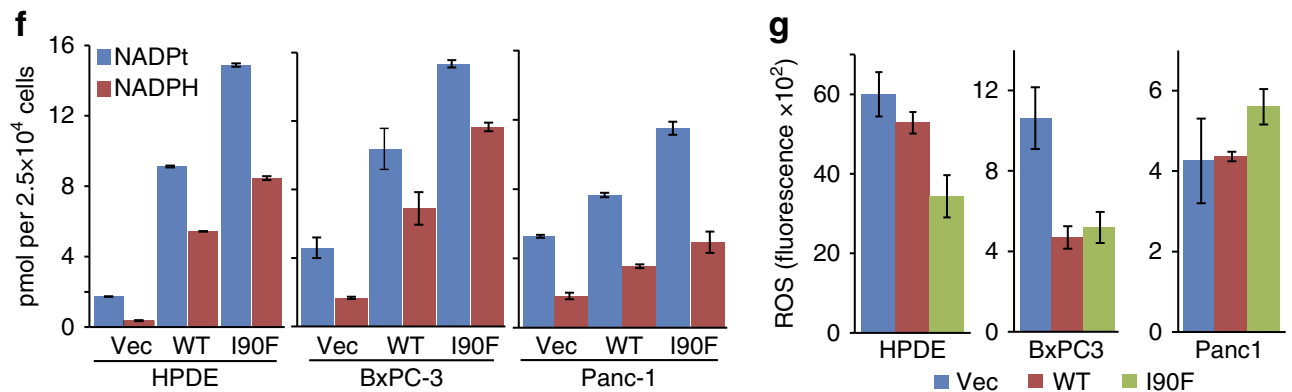

Figure 4 | NADK ${ }^{190 F}$ promotes tumorigenesis and alters redox state. (a) Kaplan-Meier survival plot for xenograft assay of HPDE-iKRASG12D cells expressing GFP $(\mathrm{Vec}), N A D K^{W T}$ or $N A D K^{190 F}$ in mice maintained Off Dox diet $(N=10$ each). $P$ value calculated by log-rank test. Complementary end point tumour volume shown at right, $P$ value by $t$-test that includes $N A D K^{W T}$ outlier. ${ }^{\star}$, outlier based on Grubb's extreme studentized deviate outlier test, $P<0.05$. (b) Protein radius of gyration plot. (c) Electrostatic potential plots comparison of wild type to I90F mutant (regions of negative potential are coloured red; regions of positive potential are coloured blue). (d) Reaction kinetics of NAD and ATP substrates for NADK WT and I90F mutant recombinant enzymes. Error bars denote s.d. of replicates $(N=3)$. (e) Immunoblot analysis of NADK expression in the indicated cell lines used for (f) quantitation of cellular NADPt/NADPH (mean value \pm s.d. of replicates, $N=3$ each) and (g) ROS through measurement of DCFDA fluorescence (mean value \pm s.d. of replicates, $N=3$ each).

(Fig. 4d) revealed an overall increase in in vitro kinase activity of recombinant $\mathrm{NADK}^{\mathrm{I} 90 \mathrm{~F}}\left(\mathrm{NAD}+: V_{\max }, 0.29 ; K_{\mathrm{m}}, 0.91\right.$. ATP: $\left.V_{\max }, 0.33 ; K_{\mathrm{m}}, 03.28\right)$ compared with NADK ${ }^{\mathrm{WT}}$ (NAD $+: V_{\max }$, $0.23 ; K_{\mathrm{m}}, 1.23$. ATP: $\left.V_{\max }, 0.25 ; K_{\mathrm{m}}, 03.77\right)$. Together, these data indicate that the I90F mutation in NADK provides GOF activity, leading to an increase in tumorigenesis and tumour growth by HPDE cells.

Given NADK's known role in phosphorylating NAD, we next quantitated total (NADPt) and reduced (NADPH) NADP in cells expressing wild-type and mutant NADK (Fig. 4e). Analysis revealed a 23 -fold increase in NADPH in HPDE cells expressing $N A D K^{I 90 F}$ compared with vector control cells, and the effects of expressing $N A D K^{I 90 F}$ were greater than wild-type (sixfold; mutant versus wild-type NADK, $P=0.0007, t$-test) consistent with GOF activity for the I90F mutation (Fig. 4f). We confirmed these results using two PDAC cell lines, BxPC-3 and Panc-1, both of which exhibited a similar trend in NADPt/NADPH levels (Fig. 4f). Consistent with increased NADPH levels, HPDE cells expressing $N A D K^{I 90 F}$ also exhibited less ROS compared with wild-type- and vector-expressing cells (Fig. 4g), suggesting that increased $N A D K$ activity elevates $\mathrm{NADPH}$, resulting in a redox shift to protect cells against oxidative stress. Wild-type and mutant NADK similarly reduced ROS in BxPC-3 cells, but did not reduce ROS in Panc-1 cells, consistent with the modest increase in NADPH (Fig. 4f) and low ROS baseline level in this cell line (Fig. 4g). 
a

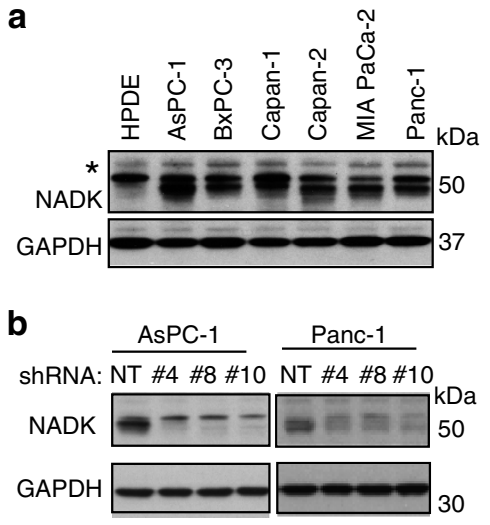

C

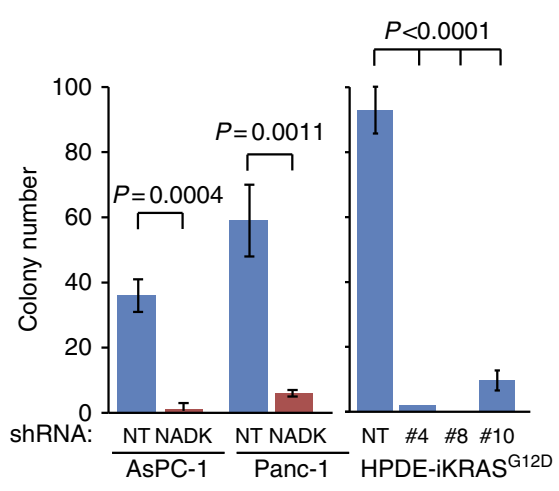

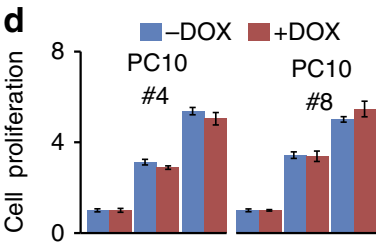

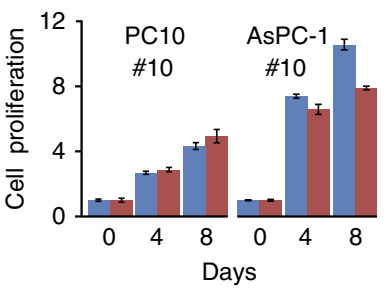

e

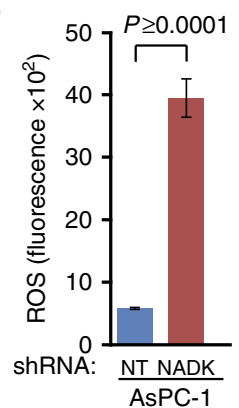

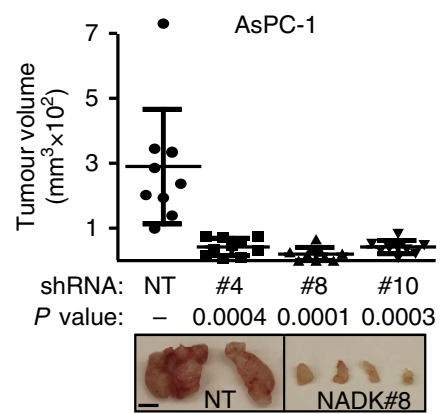
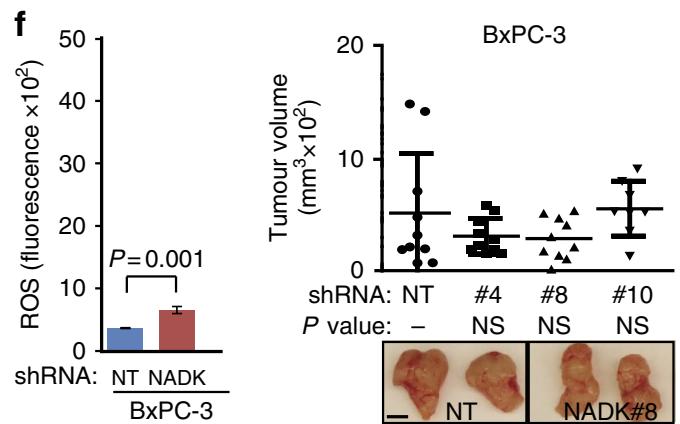

Figure 5 I NADK knockdown promotes cellular ROS and abrogates PDAC growth. (a,b) Immunoblot analysis of endogenous NADK expression in (a) HPDE and the indicated PDAC cell lines and (b) in AsPC-1 and Panc-1 cells following RNAi-mediated depletion (shNADK\#4, 8 and 10) versus NT control. * designates nonspecific band. (c) RNAi-treated cells in assessed for anchorage-independent growth. Shown are mean values $\pm s$.d. of replicates $(N=3$ each); $P$ value calculated by $t$-test. HPDE-iKRAS G12D cells were grown and plated in Dox. (d) Dox-inducible RNAi-treated PC10 fibroblasts and AcPC-1 cells assessed for proliferation in the presence or absence of Dox. Shown are mean values \pm s.d. of replicates $(N=4$ each). Full growth curves in Supplementary Fig. 6. (e,f) Impact of NADK depletion on cellular ROS (mean values \pm s.d. of replicates, $N=3$ each) and xenograft tumour growth $(N=10$ each) in $(\mathbf{e})$ AsPC-1 and (f) BxPC-3 cells. The mean tumour volumes denoted as horizontal bars, s.d. range denoted by vertical T bars. $P$-values calculated by $t$-test.

Wild-type NADK depletion attenuates PDAC growth. The I90 residue is adjacent to the NADK kinase domain, and missense mutations in NADK have been observed across other cancer types at low frequency based on mining cBioPortal (http://www.cbioportal.org/public-portal). The fact that cancer genes are often deregulated by multiple genetic and epigenetic mechanisms led us to examine expression of wild-type NADK. Consistent with this notion, immunoblot analysis of a PDAC cell line panel revealed a moderate- to high-level expression of $N A D K$ compared with primary cells (Fig. 5a). To determine whether wild-type NADK is required for PDAC cell growth, we identified three short hairpin RNAs (shRNAs; shNADK\#4, 8 and 10) whose lentiviral delivery to high NADK-expressing cell lines AsPC-1 and Panc-1 significantly depleted NADK protein expression compared with non-targeting (NT) control (Fig. 5b). Functional assessment of $N A D K$ knockdown in AsPC-1 and Panc-1 cells revealed a significant impact on anchorage-independent growth in both cell lines (Fig. 5c; 36.0- and 10.4-fold decrease, respectively), which we confirmed in HPDE-iKRAS ${ }^{G 12 D}$ cells grown in the presence of Dox (Fig. 5c; $P<0.0001, t$-test), thus indicating an ability of $N A D K$ repression to significantly abrogate $K R A S^{\mathrm{G} 12 \mathrm{D}}$-driven proliferation. Depletion of NADK in normal fibroblasts (PC10) using the same three shRNAs (shNADK\#4, 8 and 10) cloned into a Dox-inducible shRNA expression vector ${ }^{23}$ had no effect on proliferation in the presence of Dox compared with off Dox (Fig. 5d and Supplementary Fig. 6a), whereas inducible knockdown of NADK had a marked effect on AsPC-1 cancer cell proliferation by 8 days following Dox addition (Fig. $5 \mathrm{~d}$ and
Supplementary Fig. 6b) consistent with growth in soft agar (Fig. 5c). Knockdown of NADK in AsPC-1 cells also led to a marked 6.8-fold $(P<0.0001, t$-test $)$ increase in cellular ROS levels versus NT control (Fig. 5e). Importantly, this sharp increase in ROS on NADK depletion corresponded with a negative impact on AsPC-1 xenograft tumour growth (Fig. $5 \mathrm{e} ; P$ value $=0.0003, t$ test, averaged across three shRNAs versus NT), suggesting that elevated levels of ROS may be inhibitory to PDAC growth as reported previously (ref. 24 and reviewed in ref. 25). Consistent with this precept, knockdown of NADK in BxPC-3 cells led to a modest 1.8-fold increase in ROS compared with NT and did not significantly have an impact on BxPC-3 xenograft growth (Fig. 5f). Together, these data combined with the recognized importance of maintaining a redox state for PDAC tumour growth nominate $N A D K$ for additional target biology aimed at establishing its role as a novel target for drug development.

\section{Discussion}

In the era of genomics medicine, we are faced with the daunting challenge of translating genome-scale knowledge to patient care. For cancer, therapeutically targeting oncoproteins or their effectors offer great hope for improving patient outcomes. However, the use of sequencing data for drug development or re-positioning of FDA-approved drugs is challenging since bioinformatic annotation alone is insufficient to inform such efforts. The research community should therefore direct its resources towards the most promising driver candidates, 
identification of which requires robust pipelines that allow functional prioritization of the thousands of potential targets emerging from sequencing data. We describe here the infrastructure that enables functionalization of somatic mutant gene variants through their efficient construction, molecular barcoding and delivery to cancer models for in vivo tumour driver screens. While also useful for the study of low-frequency, uncharacterized tail mutations in known cancer genes, here we focus on rare mutations in genes with less or no characterized roles in cancer. Even though mutated at low frequency, the biological selection of rare mutations during malignancy, detection of activity during functional screening and initial insights into their mode-of-action may reveal new mechanisms by which tumours activate known or novel cancer signalling pathways. Much like previous efforts towards functionalizing high-frequency aberrations for clinical translation (that is, targeted therapeutics for activating mutations in EGFR, BRAF and others), identifying rare driver mutations and their mode-of-action has the potential to reveal new tumour vulnerabilities, particularly when found to activate pathways with existing therapies.

We first applied these technologies to functionally annotate PDAC gene aberrations, work that prioritized $N A D K^{I 90 F}$ for downstream functional studies and target validation. While identified in only a single PDAC patient, the mutant's GOF activity led us to investigate the role of wild-type $N A D K$, which we show is expressed at high levels in PDAC cell lines compared with normal cells and regulates redox states known to be critical for PDAC progression. Despite the importance of NADPH on redox regulation, NADK has been largely understudied in cancer and only recently been explored as a therapeutic target ${ }^{26}$. The redox state in a cell is primarily determined by the balance of NADP + I $\mathrm{NADPH}$ to maintain pools of reduced glutathione, a key pathway to reduce $\operatorname{ROS}^{27}$. ROS, which have both pro- and anti-tumorigenic effects, are often higher in cancer compared with normal cells because of mitochondrial damage, increased metabolic rates or elevated expression of oxidizing enzymes ${ }^{27-29}$. A number of studies have suggested that PDAC cells prefer low ROS (reviewed in ref. 25), rationalized based on the dual nature of ROS where low ROS levels may be growth-promoting, while high levels are cytotoxic. This notion is further supported by recent work ${ }^{24}$ reporting a novel mechanism by which KRAS rewires PDAC tumours to maximize energy production and promote $\mathrm{NADPH}$ accumulation to maintain redox state and tumour growth. This non-canonical metabolic pathway, which thus far is unique to PDAC, results in a shuttling of glutamine-derived aspartate from the mitochondria to the cytoplasm via the GOT1 transaminase to fuel NADPH production and decrease cellular $\operatorname{ROS}^{24}$. These findings along with our data lead us to hypothesize that activating NADK through mutation or increased expression may provide PDAC tumours an additional means to increase cellular $\mathrm{NADPH}$, and the interplay between GOT1-mediated glutamine utilization and NADK activity may cooperatively influence redox state and tumour growth in PDAC. More work will need to be performed to examine the possible intersection between GOT1 and NADK, as well as studies into the potential clinical impact of inhibiting these pathways for PDAC patient care.

In addition to $N A D K$, our efforts revealed potential driver activity for variants of BST1, MAGEA6, ELMOD1, WDR69, THAP10 and IGFBP5. Of these, BST1 and MAGEA6 variants exhibited robust enrichment across multiple screen tumour cores, suggesting that they exhibit potent tumour-driving activity. While additional work is needed to validate these candidate oncogenes, including assessing variant activity compared with wild type, MAGEA6 and BST1 have both been previously implicated in cancer. A recent study ${ }^{30}$ reported that MAGEA6 is overexpressed in a variety of cancers that include breast and colon, and high
MAGEA6 expression in lung squamous cell carcinoma correlates with poor overall survival. Moreover, depletion of MAGEA6 in colon and lung cancer cell lines abrogated clonogenic survival, whereas exogenous MAGEA6 expression transformed primary colonic epithelial cells ${ }^{30}$ and promoted tumour growth and metastasis in xenograft models of thyroid cancer ${ }^{31}$. BST1 metabolizes $\mathrm{NAD}^{+}$to produce cyclic ADP ribose and subsequently ADP-ribose ${ }^{32,33}$, indicating a possible role for BST1 in $\mathrm{Ca}^{2}+$ homeostasis and calcium signalling ${ }^{34}$. Notably, in monocytes the interaction between BST1 and the ECM initiates signalling cascade mediated by focal adhesion kinase and Src, leading to activation of the MAP Kinase and PI3K/Akt pathways commonly dysregulated in cancer $^{35}$. Consistent with BST1's potential role in cancer, it is overexpressed in ovarian tumours and is correlated with shorter survival ${ }^{36}$.

Future work is required to validate the activity and clinical significance of the mutations and genes identified in this study. Our focus on low-frequency driver mutations precludes identifying tumour cell lines carrying such mutations, which is particularly true for PDAC because of limited numbers of cell lines present throughout the research community; thus, it is currently not possible to determine whether silencing or complementing the described mutations would alter tumour growth by tumours/tumour cell lines harbouring those events. Future work should include engineering the identified point mutations into mice for crossing on PDAC mouse models ${ }^{37}$ driven by inducible $K R A S^{G 12 D}$. Such models would permit genetic tractability (that is, presence or absence of KRAS ${ }^{G 12 D}, T P 53$ and so on) and the proper microenvironment for assessing each mutation's ability to promote transformation, tumour initiation and progression. Nevertheless, our HiTMMoB-based screening approaches succeeded in prioritizing these events for mechanistic evaluation and secondary target biology studies to assess suitability for drug or pathway inhibitor development. When applied more broadly to other cancer types and model systems, these GOF screening technologies will reveal the highest priority targets to expedite translation of genomics data into actionable diagnostic and drug development end points.

\section{Methods}

Cell culture. All cell lines were propagated at $37^{\circ} \mathrm{C}$ and $5 \% \mathrm{CO}_{2}$ in humidified atmosphere. HPDE ${ }^{15,16}$ cells were cultured in KSFM medium (Life Technologies). Panc-1, AsPC-1, Capan-1, Capan-2, MIA PaCa-2 and BxPC-3 were purchased from American Type Culture Collection and were cultured following their recommendations. PC10 cells (provided by Dr. Penelope Bonnen, Baylor College of Medicine) were cultured in RPMI $+10 \%$ fetal bovine serum (FBS). All human cell lines were authenticated by fingerprinting using short tandem repeat testing where possible and were verified to be free of mycoplasma contamination before use. In some experiments as indicated, cells were propagated in Dox $\left(100 \mathrm{ng} \mathrm{ml}^{-1}\right)$ or glucose and glutamine-free DMEM supplemented with physiological plasma concentrations of $5 \mathrm{mM}$ glucose and $0.5 \mathrm{mM}$ L-glutamine (Corning). The Doxregulatable $K R A S^{G 12 D}$ HPDE cell line was constructed using the pInducer system $^{23}$

Immunoblot analysis. Cells were lysed using RIPA buffer (Sigma) containing Protease Inhibitor Cocktail (Sigma) with phosphatase inhibitors (Calbiochem) for separation and blotting on polyvinylidene difluoride (Millipore). The following antibodies were used for immunoblotting: NADK (Santa Cruz; sc-100347); GAPDH (Santa Cruz; sc-25778); Vinculin (Santa Cruz; sc-25336), phospho-ERK1/ 2 (Cell Signaling; 9101 S) and KRAS (Santa Cruz; sc-30). All antibodies were diluted to 1:1,000 in 1\% BSA. Full immunoblot scans are shown in Supplementary Figs 7 and 8. KRAS activity assay was performed by RAF pulldown using a Ras Activation Assay Kit (EMD-Millipore) by using $650 \mu \mathrm{g}$ of cell lysate and $10 \mu \mathrm{g}$ of RAF beads per reaction following the manufacturer's protocol.

Gene expression and RPPA analyses. RNAs extracted from the indicated cell lines cultured with or without Dox $\left(100 \mathrm{ng} \mathrm{ml}^{-1}\right.$ for $72 \mathrm{~h}$ ) were processed for hybridization on Agilent arrays (G3 Human GE 8x60K) by the Baylor College of Medicine Genome Profiling Core Facility. Array data were deposited in NCBI Gene Expression Omnibus (GEO) database (http://www.ncbi.nlm.nih.gov/geo/) under 
accession number GSE58055. RPPA was performed by the MD Anderson RPPA Core Facility using protein lysates extracted from the indicated cell lines. For each RPPA replicate, cells were washed with cold PBS twice and lysed with $100 \mu$ lysis buffer (1\% Triton X-100, $50 \mathrm{mM}$ HEPES, pH 7.4, $150 \mathrm{mM} \mathrm{NaCl}, 1.5 \mathrm{mM} \mathrm{MgCl}_{2}$, $1 \mathrm{mM}$ EGTA, $100 \mathrm{mM} \mathrm{NaF}, 10 \mathrm{mM}$ Na pyrophosphate, $1 \mathrm{mM} \mathrm{Na}_{3} \mathrm{VO}_{4}$ and $10 \%$ glycerol, containing freshly added protease and phosphatase inhibitors from Roche Applied Science Cat. \# 05056489001 and 04906837001, respectively), mixed with $4 \times$ SDS sample buffer ( $40 \%$ Glycerol, $8 \%$ SDS, $0.25 \mathrm{M}$ Tris-HCl, pH 6.8) and boiled for $5 \mathrm{~min}$ before RPPA. Two-sided homoscedastic $t$-tests (using log-transformed data) and fold changes were used to determine differentially expressed genes and proteins. Expression patterns were visualized as heatmaps using JavaTreeView. MSigDB (version 3.0) gene sets were searched using SigTerms ${ }^{38}$.

HiTMMoB and functional screening. Site-directed mutagenesis was based on modified application of megaprimer PCR ${ }^{39}$. ORF entry clones used in this study (Supplementary Table 1) were from Life Technologies or the ORFeome 8.1 (http:// horfdb.dfci.harvard.edu/). Ten nanograms of ORF DNA template was entered into a PCR reaction ${ }^{14}$ using $0.6 \mu \mathrm{M}$ modified M13F (5'-GTAAAACGACGGCC- $\left.3^{\prime}\right)$ and M13R $\left(5^{\prime}\right.$-AACAGCTATGACC- $\left.3^{\prime}\right)$ primers and $0.08 \mu \mathrm{M}$ mutation-specific primer (Supplementary Table 1) using KOD Hot Start DNA Polymerase (Novagen). Amplification parameters were as follows: $1 \times\left[95^{\circ} \mathrm{C}-4^{\prime}\right] ; 6 \times\left\{6 \times\left[95^{\circ} \mathrm{C}-45^{\prime \prime}\right.\right.$, $72^{\circ} \mathrm{C}-1$ ' $+30^{\prime \prime}$ per $1 \mathrm{~kb}$ length of ORF, $\left.95^{\circ} \mathrm{C}-45^{\prime \prime \prime}\right] ; 3 \times\left[88^{\circ} \mathrm{C}-45^{\prime \prime}, 42^{\circ} \mathrm{C}-1\right.$ ', $72^{\circ} \mathrm{C}-$ $30^{\prime \prime}$ per $1 \mathrm{~kb}$ length of ORF, $\left.\left.72^{\circ} \mathrm{C}-2^{\prime}\right]\right\}$. PCR reactions were treated with $1 \mu \mathrm{l}$ DpnI before recombination into destination vector pLenti6 V5/Dest (Life Technologies) following the manufacturer's recommendations. For barcoding, a set of BC entry clones was constructed by annealing in vitro complimentary DNA oligonucleotides containing $24 \mathrm{nt}$ BCs flanked by T3/T7 sequences and attB2r/B4 sites. Annealed oligonucleotides were subsequently recombined into modified pDONR223 by BP recombination, leading to entry clones whose $\mathrm{BCs}$ were flanked with attR2/L4 for multifragment recombineering (with ORF entry clone or mutagenesis PCR product) into pLenti6 V5/Dest BC that contains attR1 and attR4 recombination sites. STBL3 (Life Technologies) bacteria were heat-shock-transformed with the $\mathrm{BP} / \mathrm{LR}$ reaction mixtures for vector propagation. Lentivirus was produced using standard virus packaging vectors and virus protocols. For HPDE (and their derivatives) transduction, cells were infected once and allowed to recover in supplemented KSFM medium for $48 \mathrm{~h}$. For in vivo screening, $2 \times 10^{4}$ cells per virally infected HPDE line and GFP control were mixed and re-suspended in a 1:1 solution of Hank's balanced salts (Invitrogen) and Matrigel (BD Bioscience) for subcutaneous implantation into female nude animals (Harlan, Indianapolis, IN) at $5 \times 10^{5}$ total cells per site.

BC sequencing. gDNA was extracted from library-infected HPDE- $i K R A S^{G 12 D}$ cells (injected or Input) and three individual tumour cores (output) for quadruplicate and triplicate, respectively, PCR reactions to amplify the BC pools present within each sample using Platinum Super Mix (Life Technologies) and flanking primers common to each BC: $5^{\prime}$-CAATTAACCCTCACTAAAGG- $3^{\prime}$ and $5^{\prime}-$ CCGCCACTGTGCTGGATA- $3^{\prime}$ ). Amplification parameters were as follows: $1 \times\left[94^{\circ} \mathrm{C}-4^{\prime}\right] ; 35 \times\left[94^{\circ} \mathrm{C}-1^{\prime}, 54^{\circ} \mathrm{C}-1^{\prime}, 68-1^{\prime}\right] ; 1 \times\left[68^{\circ} \mathrm{C}-10^{\prime}\right]$. Each replicate PCR amplicon (178 nt) was purified for PGM library preparation, where each amplicon was ligated to unique Ion Xpress Barcode Adaptors for PGM sequencing (318 V2 Chip) following the manufacturer's recommendations. Raw data were concatenated into one 'reference' file and indexed using burrows-wheeler alignment tool ${ }^{40}$ for alignment of $\mathrm{BC}$ sequences (with parameters ' $-17-\mathrm{t} 12-\mathrm{N}-\mathrm{n} 3$ ') for counting the occurrence of each BC. BC enrichment was assessed by quantitating the number of occurrences for each BC sequence as a ratio to total number of BC reads in each sample. S.d.'s were calculated for quadruplicate and triplicate reactions for input and output samples, respectively, and were plotted as error bars on the BC enrichment graphs.

Cell assays. Soft agar colony formation assays were performed on six-well plates in triplicate. For HPDE-iKRAS and NADK shRNA-transduced cells, cells were pretreated with/without DOX $\left(100 \mathrm{ng} \mathrm{ml}^{-1}\right)$ for $24 \mathrm{~h}$ and were mixed thoroughly in cell growth medium containing $0.4 \%$ SeaKem LE agarose (Fisher) in

KSFM \pm DOX, followed by plating on bottom agarose prepared with $0.7 \%$ agarose in KSFM \pm DOX. Each well was allowed to solidify and subsequently covered in $2 \mathrm{ml} \mathrm{KSFM} \pm$ DOX, which was refreshed every 3 days. Colonies were stained with $0.05 \%(\mathrm{wt} / \mathrm{vol}$ ) iodonitrotetrazolium chloride (Sigma) and scanned at 1,200 d.p.i. using a flatbed scanner. For AsPC-1 and Panc-1, RPMI + FBS and DMEM + FBS were used instead of KSFM, respectively, followed by plating 3,000 cells in each well for each cell line as described above. For cell proliferation assays, transduced AsPC1 and PC10 cells were pretreated with DOX $\left(2 \mu \mathrm{g} \mathrm{ml}{ }^{-1}\right)$ for $48 \mathrm{~h}$, followed by plating 2,000 and 1,000 cells on White Opaque 96-well microplates in quadruplicate, respectively. Cell density was assayed with CellTiter-Glo (Promega) using a Wallac Victor2 Multilabel Counter at multiple time intervals. All data were assessed by two-tailed $t$-test calculation using Prism 4 (Graphpad).

Animal studies. Animal studies were conducted in accordance to our IACUCapproved protocol at the Baylor College of Medicine. For pancreas orthotopic injection, $1 \times 10^{6}$ HPDE-iKRAS ${ }^{G 12 D}$ cells were injected with Matrigel (BD
Biosciences; 1:1 ratio; $20 \mu$ total volume) into the tail of the pancreas in athymic mice. Mice were put On or Off Dox according to experimental design. D-luciferin (Gold Biotechnology; $1 \mathrm{~g}$ per $66 \mathrm{ml} ; 100 \mu \mathrm{l}$ per mouse) was injected via the retroorbital sinus. Mice were placed into xenogeny machine and subjected to imaging within $5 \mathrm{~min}$ for $1 \mathrm{~min}$. For xenograft tumour assays, HPDE-iKRAS ${ }^{G 12 D}$ cells transduced with GFP or ORF lentivirus (in pLenti6.3 backbone) or shRNA-treated cells were subcutaneously injected $\left(1 \times 10^{6}\right.$ cells each site $)$ into the flanks of female nude animals (athymic nude mice; CrTac:NCr-Foxn $1^{n u}$ Harlan, Indianapolis, IN) following suspension in Hank's buffer at 1:1 with Matrigel (BD Biosciences). Animals were maintained On or Off Dox diet $\left(2 \mathrm{~g} \mathrm{~kg}^{-1}\right.$; BioServ). Tissue sections were stained with haematoxylin and eosine and in some cases with CK-19 (168581-AP, Proteintech). The slides were scanned using Pannoramic 250 Flash Whole Slide Digital Scanner (3DHISTECH)

NADPt/NADPH and ROS quantification assays. NADPt/NADPH ratios were determined using a NADP/NADPH assay kit (Abcam; ab65349) as detailed in the manufacturer's protocol. Briefly, $3 \times 10^{6}$ transduced HPDE and BxPC-3 cells and $5 \times 10^{6}$ Panc- 1 cells were plated on $10-\mathrm{cm}$ plates for $24 \mathrm{~h}$, followed by replacing growth medium by nutrient-deficient DMEM (Corning; 17-207-CV) $+10 \%$ FBS $+5 \mathrm{mM}$ glucose $+0.5 \mathrm{mM}$ glutamine for $6 \mathrm{~h}$ before harvest. Overall, $2 \times 10^{6}$ cells were collected and immediately used for sample detection by and absorbance measurement using a Wallac Victor2 Multilabel Counter. For ROS measurements, $3.5 \times 10^{5}$ transduced HPDE and BxPC- 3 cells and $5 \times 10^{5}$ Panc- 1 and AsPC- 1 cells were plated on six-well plate in triplicate for $24 \mathrm{~h}$, followed by replacing growth medium by nutrient-deficient DMEM (Corning; 17-207-CV) + 10\% FBS + $5 \mathrm{mM}$ glucose $+0.5 \mathrm{mM}$ glutamine for $6 \mathrm{~h}$ before harvest. Cells were stained with $5 \mu \mathrm{M}$ $2^{\prime}, 7^{\prime}$-dichlorodihydrofluorescein diacetate (DCFDA) (Invitrogen) for $30 \mathrm{~min}$ before analysis using flow cytometry according to the manufacturer's protocol.

NADK modelling of mutant I90F. Models were built from the 3PFN.pdb (ref. 21) X-ray crystal structure of human NADK and chains A and B only (the dimer). We used the UCSF Chimera ${ }^{41}$ DockPrep tool to replace the selenomethionine (MSE) residue with methionine (MET) and replace all missing side chains in the structure using Dunbrack rotamer library ${ }^{42}$. The ATP and $\mathrm{Mg}^{2+}$ ions were docked manually based on least squares fit of the protein backbones with the X-ray crystal structure of NADK from Archaeoglobus fulgidus in complex with ATP ${ }^{43}$. We used SPDBV (Swiss PDB viewer known as 'DeepView'; ref. 44) to make the I90F mutant. All calculations were performed using the Gromacs v 4.6 .5 biomolecular dynamics software suite ${ }^{45}$. We used the Amber99SB-ILDN force field ${ }^{46}$ for the protein and the general amber force field $\left({ }^{47}\right)$ for the ATP molecule. The models were refined using steepest-descent energy minimization for both in vacuo structures using a 12$\AA$ cutoff with a shift function to smoothly decay forces from 10 to $12 \AA$ to a gradient $1000 \mathrm{~kJ} \mathrm{~mol}^{-1} \bullet \mathrm{nm}$. This method was followed by conjugating gradient minimization to a gradient of $100 \mathrm{~kJ} \mathrm{~mol}^{-1} \bullet \mathrm{nm}$. Each system was solvated in a periodic octahedral box of TIP3P water ${ }^{48}$, with $0.15 \mathrm{M} \mathrm{NaCl}$ giving a neutral system. The water was energy-minimized using steepest descents to a gradient of $1,000 \mathrm{~kJ} \mathrm{~mol}^{-1} \bullet \mathrm{nm}$ using $10 \AA$ nonbonded force cutoff and particle mesh Ewald (PME) method ${ }^{49,50}$ for long-range electrostatic interactions. We ran our simulations using a Tesla GPU card. The verlet cutoff scheme was used for all molecular dynamics simulations with a 9.0- $\AA$ short-range cutoff. The PME method was used to account for long-range electrostatic interactions with a PME order of 6 A dispersion correction for the energy and pressure was used to account for the use of a cutoff for the van der Waals interactions. The LINCS method ${ }^{51}$ was used to constrain bonds to hydrogen atoms to enable the use of a 2 -fs time step for the simulations. The velocity rescale method ${ }^{52}$ was used to maintain simulation temperature to $300 \mathrm{~K}$. The Parrinello-Rahman barostat ${ }^{53}$ was used to maintain pressure to $1 \mathrm{bar}$ for the simulations. The following procedure was used: $100 \mathrm{ps}$ Canonical ensemble (NVT) keeping protein restrained $->100 \mathrm{ps}$ Isothermalisobaric (NPT) ensemble keeping protein restrained $->60$ ns of production molecular dynamics using NPT ensemble with no restraints. Structure averages were subjected to the same energy minimization refinement protocol as the initial models. Electrostatic calculations were performed using the APBS v 1.2.1 programme $^{54}$ using $\varepsilon$ (solute) $=2.0 ; \varepsilon$ (solvent) $=78.0$; probe radius $=1.4 \AA$; $[\mathrm{NaCl}]=0.15 \mathrm{M}$ and $T=310 \mathrm{~K}$. Amber $99 \mathrm{SB}$ partial atomic charges and Amber atomic radii were used for the electrostatic calculations. We used the Pymol graphics package for all illustrations.

NADK enzymatic assay. GST-NADK WT and GST-NADK ${ }^{190 \mathrm{~F}}$ were cloned into pGEX-6 P-1 and expressed in One Shot BL21 (DE3; Life Technologies) for purification using Glutathione Agarose (Thermo Scientific) following the standard protocol. NADK and NADK ${ }^{I 90 F}$ activity was measured spectrophotometrically with a coupled assay that reduces NADP generated by NADK into detectable NADPH using the enzyme glucose-6-phosphate dehydrogenase ${ }^{55}$. Data were analysed using GraphPad Prism 6 by means of Michaelis-Menten kinetics ${ }^{56}$.

NADK knockdown studies. NADK knockdown studies were performed using pLKO-shGFP (NT) or each of the following shRNAs available from the RNAi Consortium (TRC; Broad Institute): TRCN0000037702 (shNADK\#4), TRCN0000199808 (shNADK\#8), TRCN0000199040 (shNADK\#10). shRNA target 
sequences are as follows: shGFP (NT): 5'-CAAGCTGACCCTGAAGTTCAT-3'; shNADK\#4: $5^{\prime}$-GCATCAGCATCACTACCTCAT-3'; shNADK\#8: $5^{\prime}$-GATGACA TTTCCAATCAGATA-3'; shNADK\#10: $5^{\prime}$-GAATAAGGAATTGAGTCCAGA-3'

\section{References}

1. Moody, S. E., Boehm, J. S., Barbie, D. A. \& Hahn, W. C. Functional genomics and cancer drug target discovery. Curr. Opin. Mol. Ther. 12, 284-293 (2010).

2. Mohr, S. E., Smith, J. A., Shamu, C. E., Neumuller, R. A. \& Perrimon, N. RNAi screening comes of age: improved techniques and complementary approaches. Nat. Rev. Mol. Cell Biol. 15, 591-600 (2014).

3. Dunn, G. P. et al. In vivo multiplexed interrogation of amplified genes identifies GAB2 as an ovarian cancer oncogene. Proc. Natl Acad. Sci. USA 111, 1102-1107 (2014)

4. Wang, Y. et al. MELK is an oncogenic kinase essential for mitotic progression in basal-like breast cancer cells. eLife 3, e01763 (2014).

5. Johannessen, C. M. et al. A melanocyte lineage program confers resistance to MAP kinase pathway inhibition. Nature 504, 138-142 (2013).

6. Shrestha, Y. et al. PAK1 is a breast cancer oncogene that coordinately activates MAPK and MET signaling. Oncogene 31, 3397-3408 (2012).

7. Boehm, J. S. et al. Integrative genomic approaches identify IKBKE as a breast cancer oncogene. Cell 129, 1065-1079 (2007).

8. Pavlova, N. N. et al. A role for PVRL4-driven cell-cell interactions in tumorigenesis. eLife 2, e00358 (2013).

9. Rual, J. F. et al. Human ORFeome version 1.1: a platform for reverse proteomics. Genome Res. 14, 2128-2135 (2004).

10. Lamesch, P. et al. hORFeome v3.1: a resource of human open reading frames representing over 10000 human genes. Genomics 89, 307-315 (2007).

11. Yang, X. et al. A public genome-scale lentiviral expression library of human ORFs. Nat. Methods 8, 659-661 (2011).

12. Temple, G. et al. The completion of the Mammalian Gene Collection (MGC). Genome Res. 19, 2324-2333 (2009).

13. Biankin, A. V. et al. Pancreatic cancer genomes reveal aberrations in axon guidance pathway genes. Nature 491, 399-405 (2012).

14. Wu, W., Jia, Z., Liu, P., Xie, Z. \& Wei, Q. A novel PCR strategy for high-efficiency, automated site-directed mutagenesis. Nucleic Acids Res. 33, e110 (2005).

15. Furukawa, T. et al. Long-term culture and immortalization of epithelial cells from normal adult human pancreatic ducts transfected by the E6E7 gene of human papilloma virus 16. Am. J. Pathol. 148, 1763-1770 (1996).

16. Ouyang, H. et al. Immortal human pancreatic duct epithelial cell lines with near normal genotype and phenotype. Am. J. Pathol. 157, 1623-1631 (2000).

17. Kuilman, T., Michaloglou, C., Mooi, W. J. \& Peeper, D. S. The essence of senescence. Genes Dev. 24, 2463-2479 (2010).

18. Singh, A. et al. A gene expression signature associated with "K-Ras addiction" reveals regulators of EMT and tumor cell survival. Cancer Cell 15, 489-500 (2009).

19. Pollak, N., Dolle, C. \& Ziegler, M. The power to reduce: pyridine nucleotides-small molecules with a multitude of functions. Biochem. J. 402, 205-218 (2007).

20. Sousa, C. M. \& Kimmelman, A. C. The complex landscape of pancreatic cancer metabolism. Carcinogenesis 35, 1441-1450 (2014).

21. Wang, H. et al. Crystal structure of human NAD kinase. RCSB PDB (2011).

22. Rod, T. H., Radkiewicz, J. L. \& Brooks, 3rd C. L. Correlated motion and the effect of distal mutations in dihydrofolate reductase. Proc. Natl Acad. Sci. USA 100, 6980-6985 (2003).

23. Meerbrey, K. L. et al. The pINDUCER lentiviral toolkit for inducible RNA interference in vitro and in vivo. Proc. Natl Acad. Sci. USA 108, 3665-3670 (2011).

24. Son, J. et al. Glutamine supports pancreatic cancer growth through a KRASregulated metabolic pathway. Nature 496, 101-105 (2013).

25. Kong, B., Qia, C., Erkan, M., Kleeff, J. \& Michalski, C. W. Overview on how oncogenic Kras promotes pancreatic carcinogenesis by inducing low intracellular ROS levels. Front. Physiol. 4, 246 (2013).

26. Hsieh, Y. C. et al. Enhanced degradation of dihydrofolate reductase through inhibition of NAD kinase by nicotinamide analogs. Mol. Pharmacol. 83, 339-353 (2013)

27. Fruehauf, J. P. \& Meyskens, Jr F. L. Reactive oxygen species: a breath of life or death? Clin. Cancer Res. 13, 789-794 (2007).

28. Cui, X. Reactive oxygen species: the achilles' heel of cancer cells? Antioxid. Redox Signal. 16, 1212-1214 (2012).

29. Liou, G. Y. \& Storz, P. Reactive oxygen species in cancer. Free Radic. Res. 44, 479-496 (2010)

30. Pineda, C. T. et al. Degradation of AMPK by a cancer-specific ubiquitin ligase. Cell 160, 715-728 (2015).

31. Liu, W., Cheng, S., Asa, S. L. \& Ezzat, S. The melanoma-associated antigen A3 mediates fibronectin-controlled cancer progression and metastasis. Cancer Res. 68, 8104-8112 (2008).

32. Hirata, Y. et al. ADP ribosyl cyclase activity of a novel bone marrow stromal cell surface molecule, BST-1. FEBS Lett. 356, 244-248 (1994).
33. Kajimoto, Y. et al. Pancreatic islet cells express BST-1, a CD38-like surface molecule having ADP-ribosyl cyclase activity. Biochem. Biophys. Res. Commun. 219, 941-946 (1996).

34. Lee, H. C. Cyclic ADP-ribose and nicotinic acid adenine dinucleotide phosphate (NAADP) as messengers for calcium mobilization. J. Biol. Chem. 287, 31633-31640 (2012).

35. Lo Buono, N. et al. The CD157-integrin partnership controls transendothelial migration and adhesion of human monocytes. J. Biol. Chem. 286, 18681-18691 (2011).

36. Ortolan, E. et al. Functional role and prognostic significance of CD157 in ovarian carcinoma. J. Natl Cancer Inst. 102, 1160-1177 (2010).

37. Hingorani, S. R. et al. Preinvasive and invasive ductal pancreatic cancer and its early detection in the mouse. Cancer Cell 4, 437-450 (2003).

38. Creighton, C. J., Nagaraja, A. K., Hanash, S. M., Matzuk, M. M. \& Gunaratne, P. H. A bioinformatics tool for linking gene expression profiling results with public databases of microRNA target predictions. RNA 14, 2290-2296 (2008).

39. Kammann, M., Laufs, J., Schell, J. \& Gronenborn, B. Rapid insertional mutagenesis of DNA by polymerase chain reaction (PCR). Nucleic Acids Res. 17, 5404 (1989).

40. Li, H. \& Durbin, R. Fast and accurate short read alignment with BurrowsWheeler transform. Bioinformatics 25, 1754-1760 (2009).

41. Pettersen, E. F. et al. UCSF Chimera--a visualization system for exploratory research and analysis. J. Comput. Chem. 25, 1605-1612 (2004).

42. Dunbrack, Jr R. L. Rotamer libraries in the 21st century. Curr. Opin. Struct. Biol. 12, 431-440 (2002).

43. Liu, J. et al. Crystal structures of an NAD kinase from Archaeoglobus fulgidus in complex with ATP, NAD, or NADP. J. Mol. Biol. 354, 289-303 (2005).

44. Guex, N. \& Peitsch, M. C. SWISS-MODEL and the Swiss-PdbViewer: an environment for comparative protein modeling. Electrophoresis 18, 2714-2723 (1997).

45. Pronk, S. et al. GROMACS 4.5: a high-throughput and highly parallel open source molecular simulation toolkit. Bioinformatics 29, 845-854 (2013).

46. Lindorff-Larsen, K. et al. Improved side-chain torsion potentials for the Amber ff99SB protein force field. Proteins 78, 1950-1958 (2010).

47. Wang, J., Wolf, R. M., Caldwell, J. W., Kollman, P. A. \& Case, D. A. Development and testing of a general amber force field. J. Comput. Chem. 25, 1157-1174 (2004).

48. Jorgensen, W. L., Chandrasekhar, J., Madura, J. D., Impey, R. W. \& Klein, M. L. Comparison of simple potential functions for simulating liquid water. J. Chem. Phys. 79, 926-935 (1983).

49. Darden, T., York, D. \& Pedersen, L. Particle mesh Ewald: an Nślog(N) method for Ewald sums in large systems. J. Chem. Phys. 98, 10089-10092 (1993).

50. Essmann, U. et al. A smooth particle mesh Ewald method. J. Chem. Phys. 103, 8577-8593 (1995).

51. Hess, B., Bekker, H., Berendsen, H. J. C. \& Fraaije, J. G. E. M. LINCS: A linear constraint solver for molecular simulations. J. Comput. Chem. 18, 1463-1472 (1997).

52. Bussi, G., Donadio, D. \& Parrinello, M. Canonical sampling through velocity rescaling. J. Chem. Phys. 126, 014101 (2007).

53. Parrinello, M. \& Rahman, A. Polymorphic transitions in single crystals: a new molecular dynamics method. J. Appl. Phys. 52, 7182-7190 (1981).

54. Baker, N. A., Sept, D., Joseph, S., Holst, M. J. \& McCammon, J. A. Electrostatics of nanosystems: application to microtubules and the ribosome. Proc. Natl Acad. Sci. USA 98, 10037-10041 (2001).

55. Lerner, F., Niere, M., Ludwig, A. \& Ziegler, M. Structural and functional characterization of human NAD kinase. Biochem. Biophys. Res. Commun. 288, 69-74 (2001).

56. Michaelis, L. \& Menten, M. M. The kinetics of invertin action. 1913. FEBS Lett. 587, 2712-2720 (2013)

\section{Acknowledgements}

We thank Kristen Karlin and Trey Westbrook for providing and consulting on use of the pINDUCER vector system ${ }^{23}$ used to express KRAS ${ }^{G 12 D}$. This project was supported in part by the Genomic and RNA Profiling Core at Baylor College of Medicine with funding from the NIH/NCI grant (P30CA125123). This work was supported by the Cancer Prevention and Research Institute of Texas (CPRIT; RP120046), Lustgarten Foundation (RFP-B-042) and the 2014 Pancreatic Cancer Action Network-AACR Career Development Award (14-20-25-SCOT) by funding to K.L.S. This work was also supported by the NIH (UO1CA168394) by funding to K.L.S and G.B.M. T.D. was supported by a training grant from The Cullen Foundation. H.L. was supported by a training grant from CPRIT (RP140102). J.B.D. was supported by the American Cancer Society Joe and Jessie Crump Postdoctoral Fellowship. M.L. was supported by the NIH (R01CA138701).

\section{Author contributions}

Y.H.T. and T.D. designed and performed experiments, produced figures and wrote the manuscript. P.T. designed kinase experiments and produced figures. J.R.B. supported 
kinase experiments. H.L., J.-W.O., N.N., R.M., J.B.D. and A.D. performed experiments. M.-C.G. provided patient tumour sequencing data. M.L., Q.C., H.Y. and M.I. performed pathology analysis. G.B.M. supported RPPA experiment. F.C. and C.J.C analysed microarray and RPPA data, and produced figures. J.K. performed computational modelling for NADK molecule and produced figures. M.E. performed the NGS run. K.E. and G.B.M. supported the NGS experiment. Z.C. and K.C. analysed the NGS data for BC enrichment analysis. K.L.S. supervised the study, designed experiments and wrote the manuscript.

\section{Additional information}

Accession codes: The microarray data have been deposited in the NCBI GEO database under accession code GSE58055.

Supplementary Information accompanies this paper at http://www.nature.com/ naturecommunications

Competing financial interests: The authors declare no competing financial interests.

Reprints and permission information is available online at http://npg.nature.com/ reprintsandpermissions/

How to cite this article: Tsang, Y. H. et al. Functional annotation of rare gene aberration drivers of pancreatic cancer. Nat. Commun. 7:10500 doi: 10.1038/ncomms10500 (2016).

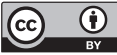

This work is licensed under a Creative Commons Attribution 4.0 International License. The images or other third party material in this article are included in the article's Creative Commons license, unless indicated otherwise in the credit line; if the material is not included under the Creative Commons license, users will need to obtain permission from the license holder to reproduce the material. To view a copy of this license, visit http://creativecommons.org/licenses/by/4.0/ 